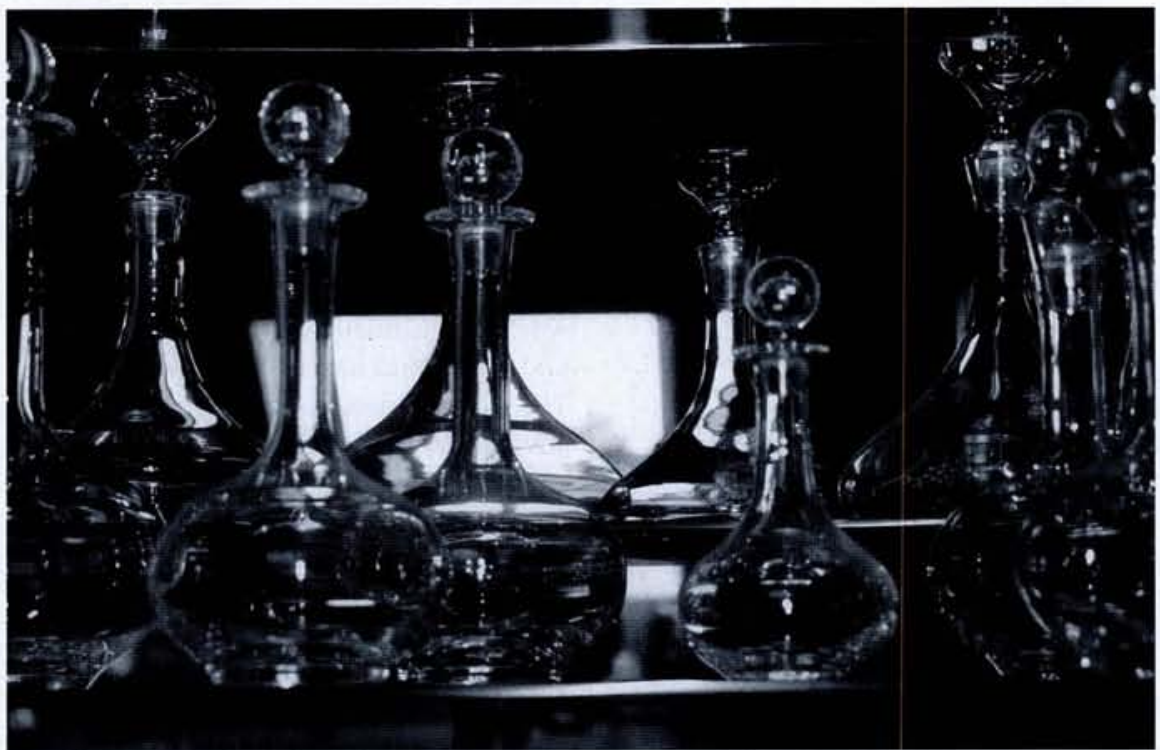

\section{A Random Walk in Search of the Glass Transition}

lan Campbell and Pierre-Olivier Mari, Université Paris-Sud

Angel Alegría and Juan Colmenero, UPV/EHU (The University of the Basque Country)

Thousands of tonnes of glass and glassy plastics are produced every day.

The freezing process itself, as molten glass hardens, is understood by craftsmen and factory managers well enough for glass to have become an indispensable material in everyday life. But freezing has not yet yielded fully to the understanding a physicist needs

For thousands of years man has made glass and learned to shape it and colour it exquisitely. Some ancient civilisations invested its manufacture with ritual and mystery. Today, the mystery persists in the challenges it poses to modern science. The "inextricably entangled skein" of atoms in a glass (as John Tyndall put it) is not easily captured and expressed in mathematical form, far less so the complicated cooperative slowing down that brings the glass forming liquid to the solid state.

At the heart of the difficulty is the glass transition, which takes place as the molten glass (called the glass former) is cooled. It is quite unlike the formation of a crystalline solid: concepts of thermodynamics which are tested and trusted for crystals become debatable with glass.

Phase transitions are usually classed as first or second order. The formation of ice crystals from water is first order, meaning that the liquid changes discontinuously into something very different. But certain liquids (including water in extreme circumstances) do not solidify this way. Instead, the atoms retain much of the disorder inherent in the liquid state, and just settle down to a quiet life in a static arrangement. This glass transition has remained poorly understood, even to the extent that its status as a thermodynamic transition has been questioned. It is a continuous transition, like a second order transition, but in contrast to what happens at second order transitions, there is no simple low temperature state being formed which can be recognized as being 'ordered'.

\section{Spin Doctors Clink Glasses}

In a glass transition the molecular movements slow down progressively until everything in the system has become frozen for all practical time scales. There is no danger of the glass in your hand flowing like the whisky it contains before you have time to drink, although old win-
"To many persons here present a block of ice may seem of no more interest and beauty than a block of glass; but in reality it bears the same relation to glass that orchestral harmony does to the cries of the market place. The ice is music, the glass is noise, the ice is order, the glass is confusion. In the glass, molecular forces constitute an inextricably entangled skein."

\section{John Tyndall 1863}

dows are sometimes slightly distorted due to flow. Looking closely at the glass transition, we find that the viscosity of the glass forming liquid diverges as a characteristic temperature is reached. In other words, the relaxation time for atomic movements becomes indefinitely large. Moreover, it does so in a peculiar manner.

Close to the glass transition relaxations are slow and their time dependence does not take the exponential form that is almost ubiquitous in the physical world. This trait is shared by magnetic 'spin glasses' (local moments with random interactions between them).

The spin glass has a magnetic transition analogous to the solidification of a glass. At high temperatures the local spins are reorienting all the time; then below a characteristic temperature each spin gels to a fixed orientation. However, the spins at low temperatures appear to be just as random as they would in a snapshot of the high temperature paramagnet. A compari- 
son between these two types of disordered systems suggests a generic description of the freezing process. It is rather abstract, but may offer a new route towards a fuller understanding of the transition itself.

The favourite spin glass systems of the laboratory are dilute alloys where the dilute component is magnetic, such as $\mathrm{CuMn}$ or AuFe (magnetic $\mathrm{Mn}$ and Fe make up only a few per cent of the atoms). In these alloys, the interactions between the magnetic spins through the conduction electrons are effectively random and oscillate in sign (as a function of distance between spins), so no simple type of order (ferromagnetism or antiferromagetism) can satisfy all interactions simultaneously. But at low temperature the spins are frozen into orientations which do their best to minimise the magnetic energy locally; there are, however, vast numbers of possible global ground states (or low energy metastable states) which all minimise the total energy of the whole system of spins almost equally well. And it is because these frozen-in spin configurations have no obvious magnetic structure that spin glasses were given their name in the first place.

Numerical work has concentrated on the Edwards-Anderson spin glass, an idealized model system. This consists of Ising
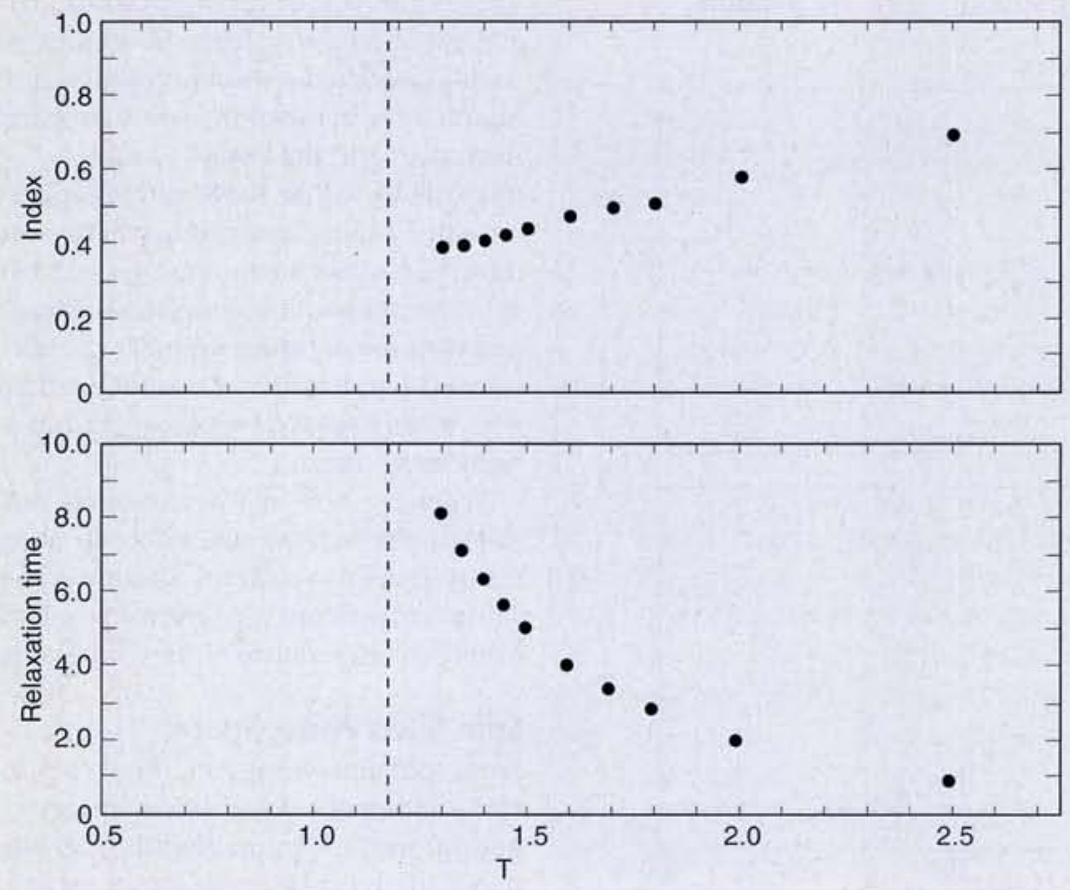

Fig 1 Changing phases: these graphs show the fall into a frozen state. The data plotted come from a computer simulation of a model spin glass. At each temperature $T$, local magnetization in the spin glass has a certain relaxation time, and a non-exponentiality index (which is $\beta$ in the stretched exponential see box). The dotted vertical line is the critical temperature. As the temperature falls relaxation time gets longer and longer, and diverges at the critical temperature; the spin glass freezes. The parameter $\beta$ (index) extrapolates to about $1 / 3$ at this temperature size scaling' techniques: simulations are made on samples of different linear sizes $L$ and scaling rules are used to estimate the transition temperature. Consistency between the two methods ensures that $T_{g}$ is a true thermodynamic transition temperature. As $T$ approaches $T_{g}, \beta$ decreases and tends toward a value near to $1 / 3$ (figure 1 top). This behaviour turns out to be characteristic of Ising spin glasses in general; even for systems of different finite dimensions and with different sets of interactions, stretched exponential functions fit the relaxation behaviour well, and always with $\beta$ showing similar temperature dependence at the approach to $T_{g}$.

\section{Polymer Glasses}

We now turn to a very different case, that of the dielectric relaxation of the local electric dipoles in a polymer glass. We choose as an example PVME (poly(vinyl methyl ether)), one of the large family of materials known familiarly as 'plastics'. Experiments measuring the relaxation function using different techniques cover a wide range of time scales - fifteen orders of magnitude in time. The relaxation throughout is fitted very satisfactori-

\section{The Stretched Exponential} $\exp \left[-(t / \tau)^{\beta}\right]$

\section{Creeping Silk Threads}

The stretched exponential function was first introduced phenomenologically by R. Kohlrausch in 1854 to fit his experimental data on the decay of the electric polarisation of Leiden jars, which are primitive capacitors. He found that the relaxation was not exponential and that the rate of relaxation effectively slowed down as time progressed. The function he used fitted the data well. His son used the same function a few years later for the creep behaviour of silk threads that were used for galvanometer needle suspensions. Rediscovered by Williams and Watts in 1970, this function has become ubiquitous in the analysis of relaxation data in glassy systems. The parameter $\beta$ expresses the non-exponential character of the relaxation; if $\beta$ is 1 the relaxation is purely exponential with a single characteristic time, but if $\beta$ is small the relaxation spectrum extends over a wide range of time scales. The decay function corresponds to a very specific spectrum of relaxation times. 


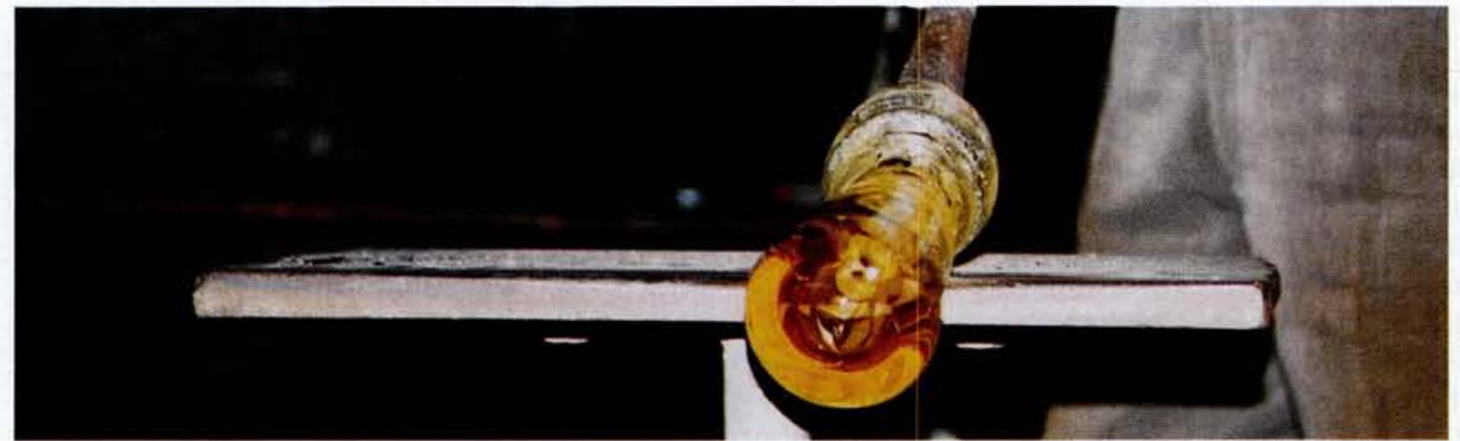

Glass blowing artisans know well enough how glass behaves. Left, a small decanter being rolled. The glass blowing itself takes only two minutes from the semi-molten glass blob to the finished decanter ready to harden slowly in the oven ly by stretched exponential functions. It can be seen in figure 2 that the relaxation time scale $\tau(T)$ is tending to diverge at a temperature somewhat below $250 \mathrm{~K}$, and that simultaneously the Kohlrausch parameter $\beta$ is heading towards a value near $1 / 3$ at the temperature where $\tau$ diverges. Many other polymer glasses have been found to show essentially the same behaviour. The data shown in figures 1 and 2 are strikingly similar, which is remarkable seeing as the two systems are so different - the spin glass results are for an artificial numerical magnetic system with disorder (the random interactions) present at all temperatures. The polymer glass data are experimental results for dielectric relaxation in a system where the glassyness arises spontaneously because of steric hindrance and a lack of regularity of the polymer chains. The two systems have nothing in common, except that they both have glass transitions. But in this way evidence accumulates of behaviour common to many glass transitions, and we can thus look for a unifying interpretation. In doing so, we use the concept of a phase space.

\section{Phase Space}

The global phase space of a system consists of all its possible configurations or microscopic instantaneous states. For example, the phase space for $N$ atoms of an ideal gas is a space of dimension $6 \mathrm{~N}$ (six because there are three space coordinates and three velocity components for each atom) and is discussed in all thermodynamics textbooks.

The phase space of a set of Ising spins is simpler. For a system consisting of three Ising spins all the eight possible
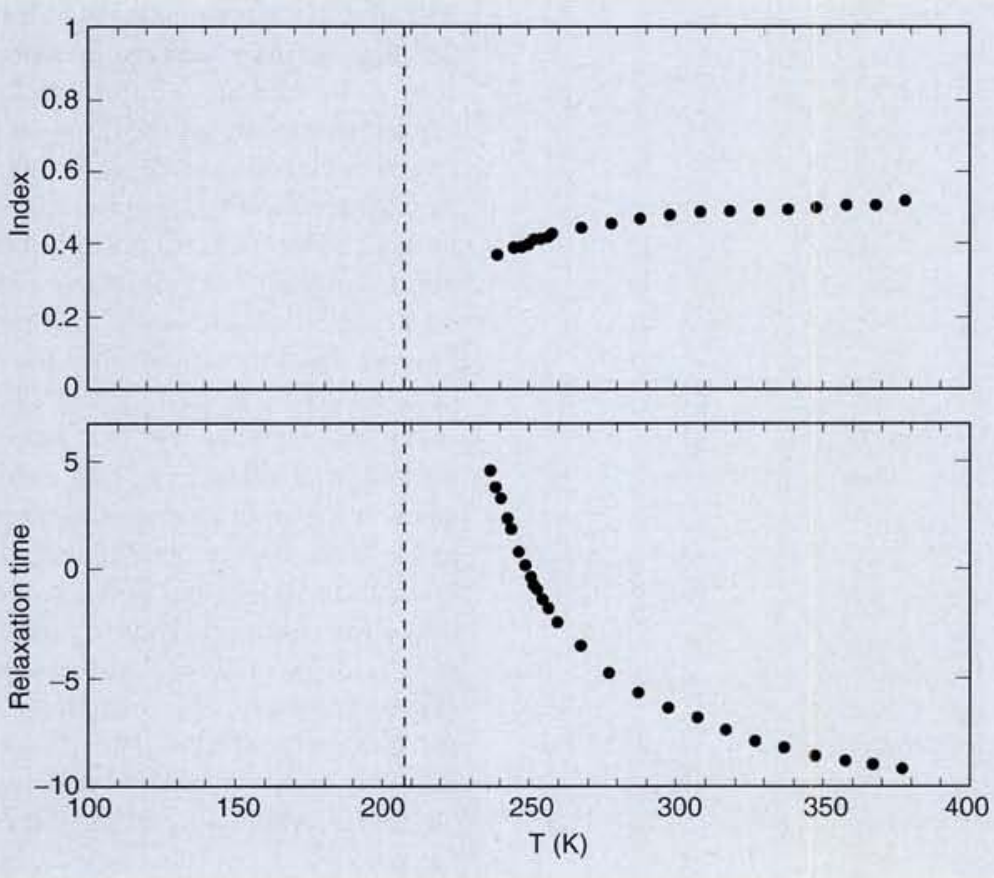

Fig 2 Relaxation in a plastic polymer. As in figure 1 relaxation time diverges at about the temperature of the dotted line: as the temperature is reduced the viscosity of the molten polymer becomes larger and larger, until the frozen glass is formed. Again, the parameter $\beta$ (index) extrapolates to about $1 / 3$ at this temperature microstates can be shown mapped onto the corners of a cube (figure 3). For an $\mathrm{N}$ spin Ising system the configurations can be mapped exactly onto the $2^{N}$ corners of an $\mathrm{N}$-dimensional hypercube; this is the global phase space. Also, as one can check from looking at figure 3, turning over one spin is equivalent to a step from one microstate (ie one corner) to a near neighbour microstate. This remains true whatever $N$ is.

As time progresses spins will flip or, in other words, the point representing the instantaneous state of the system will wander from microstate to another microstate on the hypercube. When there are interactions between the spins, different microstates will have different energies. Thermodynamics tell us that at low temperature only the low energy microstates will be thermodynamically accessible, so the system will wander within this restricted set of microstates. If the interactions between the spins are purely ferromagnetic, the lowest energy microstates will be those with all spins up or with all spins down; at low temperature the set of accessible microstates will be split up into two disconnected clusters: one with mostly spins up (near the bottom left-hand corner of the cube) and one with mostly spins down (near the topright hand corner).

When the system is heated, more and more higher energy states become accessible until the two clusters coalesce at the Curie temperature (the critical point: the ordering temperature of the ferromagnet).

\section{Spin Glass Phase Space}

For a spin glass things are more complicated with many possible low-energy ground states. Suppose that the thermodynamically accessible configurations at a given temperature are distributed completely at random over the hypercube. This is too extreme an hypothesis for a real system but it allows us to make progress. The problem is now mapped 


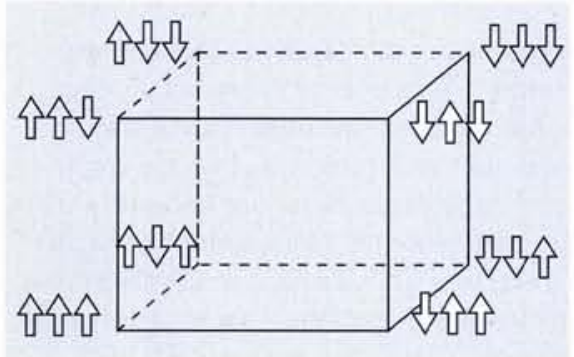

Fig 3 The phase space for a three spin lsing system. Ising spins can be either up or down, so each corner of the cube, with the three spin components in a different configuration, represents the system in a different state. Turning over one spin corresponds to moving to a neighbouring configuration. Such a geometrical model can help to explain phase transitions

onto a purely geometrical representation.

Imagine a high-dimensional hypercube with probability $p$ that any individual microstate is accessible. All the other corners are inaccessible ( $p$ plays the role of the temperature; for low $p$, fewer configurations are accessible). For this hypercube geometry it has been proved mathematically that below a critical $p$ value, $p_{c}$, there occurs the closed-space analogue of a percolation transition (see box The
Percolation Transition). Thus for $p>p c$ there is a giant cluster of linked corners while for $p<p_{c}$ there exist only isolated small clusters.

\section{The Forgetful Ant}

To see what the phase space morphology represents in terms of relaxation, we must imagine an ant representing the instantaneous configuration of the spins wandering in phase space. We set the ant off on a random walk on the giant cluster for a hypercube with sites occupied with probability $p$. As time increases, the ant moves around the sites and loses memory of its starting point, the initial configuration. It turns out that on the hypercube, the equivalent of a random walk with exponent $\beta$ in an Euclidean space (see box The Percolation Transition) is a memory function in the form of a stretched exponential with Kohlrausch exponent $\beta$. At high $p$ relaxation is rapid and exponential $(\beta=1)$; as $p$ tends towards $p_{c}$ the randomwalk memory-function time scale tends to diverge, and the Kohlrausch exponent $\beta$ tends towards $1 / 3$.

\section{The Percolation Transition}

\section{The Wandering Ant}

Take a regular Euclidean lattice of dimension $d$ having its lattice points occupied at random with probability $p$. If $p$ is greater than a critical value $p_{c}$ (which depends on the dimension and the type of lattice) then there is always a continuous path to go from one side of the lattice to the other using occupied lattice points as stepping stones. But if $p$ is less than $p_{c}$ then no such path exists. When $p$ is exactly $p_{c}$, this is the percolation transition and the cluster of linked points (the percolation cluster) has special fractal properties.

One such property concerns random walks. If an ant performs a random walk on the percolation cluster, the average of the square of the distance $L$ from its starting point increases with time $t$ as (average) $L^{2} \propto t^{\beta}$, rather than $L^{2} \propto t$ as in a conventional random walk. This is because on the percolation cluster the ant wastes time going along dead ends. If the dimension is greater than 6 , beta is equal to precisely $1 / 3$.

\section{Random Walks}

'She went on,"Would you tell me please which way I ought to go from here ?"

"That depends a good deal on where want to get to," said the Cat. "I don't much care where-" said Alice.

"Then it doesn't matter which way you go," said the Cat. "-as long as I get somewhere," Alice added as an explanation. "Oh, you are sure to do that," said the Cat, "if you only walk long enough."' Lewis Carroll Alice in Wonderland

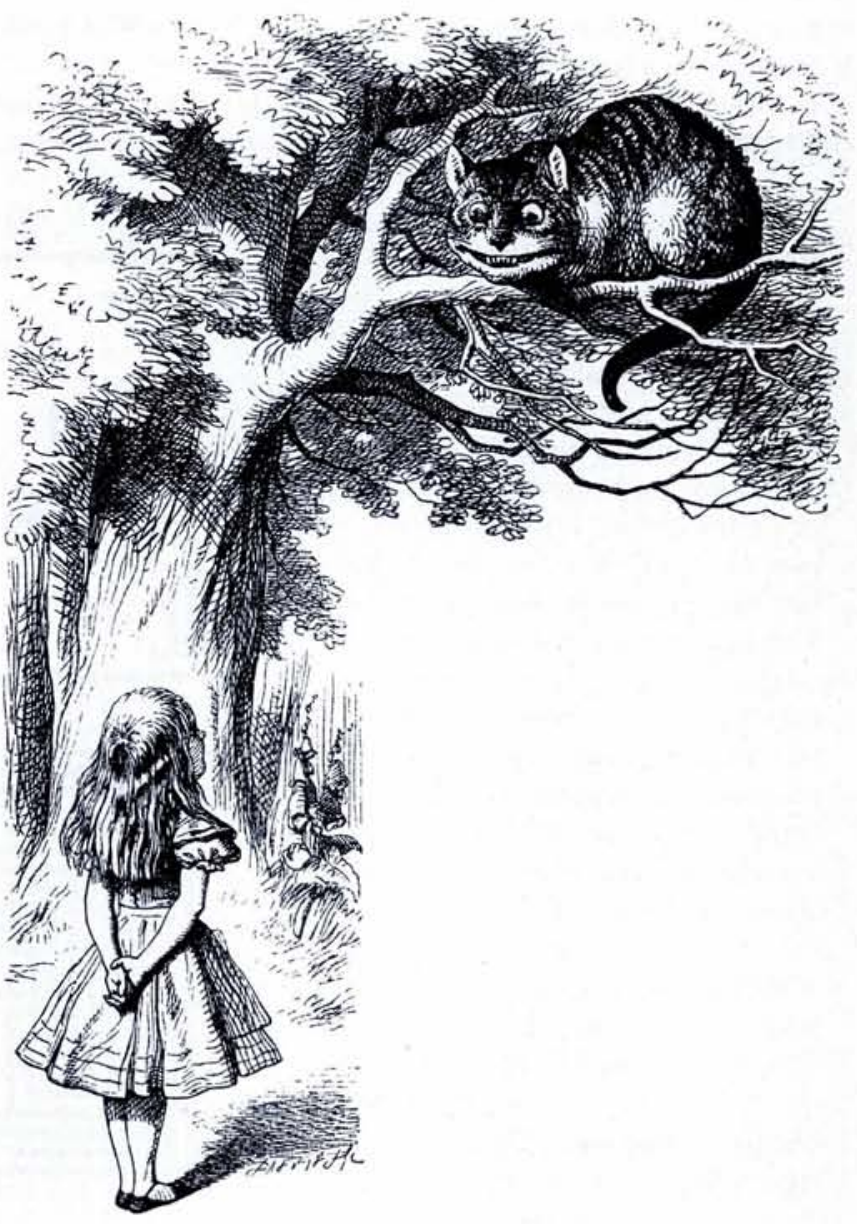


We conclude that the observed stretched-exponential relaxation behaviour in an Ising spin glass signifies that its phase space resembles the randomly occupied hypercube. We also conclude, and this is the important bit, that the spin glass transition can be thought of as a percolation transition in phase space.

\section{Marooned on a Cluster}

Glass transition in the real world: the phase space of a polymer glass former is much more difficult to imagine, but a phase space there must be. We can argue from the similarity between the relaxation behaviours of the idealized spin glass and of the polymer glass that the overall morphology of the glass phase space as the critical temperature is approached is of the same type. What concrete image does this give of the glass transition?

Below the temperature of the transition the equilibrium phase space is an archipelago of isolated clusters of configurations in phase space; the system would stay marooned on one particular cluster (different after each quench) and so would behave as a solid. In contrast, well above the transition the system would completely lose memory in a short time because it can sample enough of phase space, and so it can be considered to be a liquid.

In this approach, as the material is cooled towards the freezing temperature, phase space becomes sparse and fractallike; the system takes longer and longer to explore enough of phase space to decide if it is still above the glass transition or not. The relaxation is slow and takes up the stretched exponential form (see box Transition: the Explanation).

From $\tau(T)$ data like those in figure 2, the critical temperature, corresponding to the onset of infinitely slow relaxation, can be estimated, but by $10 \mathrm{~K}$ or so above this temperature the time scale is already weeks; in practice it is impossible to cool down slowly enough to stay in equilibrium right down to the critical temperature. A similar problem occurs for simulations in large spin glass samples (with computer time replacing real time) but it can be bypassed by using scaling rules on small samples; it is certain that the spin glass has a bona fide transition in 3 dimensions and above. The polymer glass relaxation behaves in an almost identical way to that of the spin glass in all the reduced temperature regions where equilibrium can still be achieved in both systems. Hence, it can be concluded that the polymer glass and the spin glass are heading towards true transitions of the same type.

\section{Life is complicated}

We seem finally to have an explanation of the strange stretched-exponential relaxation which is always seen above the glass temperature, in terms of a fractal-like phase space morphology. This method could in principle be extended to other contexts where the dynamics of complex systems are involved, such as the stock exchange fluctuations (see opposite page) or virus genetics. Life is complicated, as are most real materials, so we may expect to see glassy transition phenomena cropping up in many unexpected places.

The editors would like to thank co-author Ian Campbell, who is a Director of Research of CNRS, for his help with this article

Further reading

John A. Mydosh Spin Glasses: An Experimental Introduction; Taylor and Francis, London, 1993 an experimental approach to spin glass physics K.H. Fischer and J.A. Hertz Spin Glasses; Cambridge University Press, 1991 a theoretical viewpoint

John D. Ferry Viscoclastic Properties of Polymers; John Wiley and sons,

New York, 1980 a classic on polymer dynamics

Gret Strobl The Physics of Polymers: Concepts for Understanding their Structures and Behaviour; SpringerVerlag, Berlin, 1996 an introduction to polymer physics
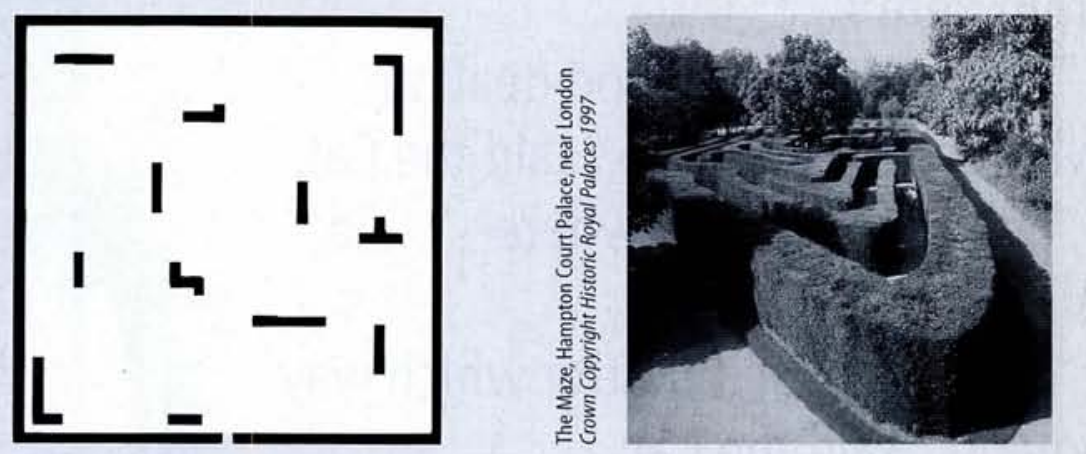

\section{A Maze in Transition}

A maze helps to illustrate the idea of a transition (and a complex phase space). Imagine a maze for which gardeners are gradually putting in more and more hedges. If the gardeners have so far only planted a few hedges (left top) it is easy to move around high temperature, fast exponential relaxation. When the maze is finished (left bottom) you have to follow a very tortuous path to get from once place to another, with the risk of going up blind alleys. This is slow stretched exponential relaxation just above the freezing temperature. However, if the gardeners went on planting bushes (right bottom) there would be a critical point beyond which there would be so many hedges that you would always get stuck in one part of the maze. This is the frozen state.

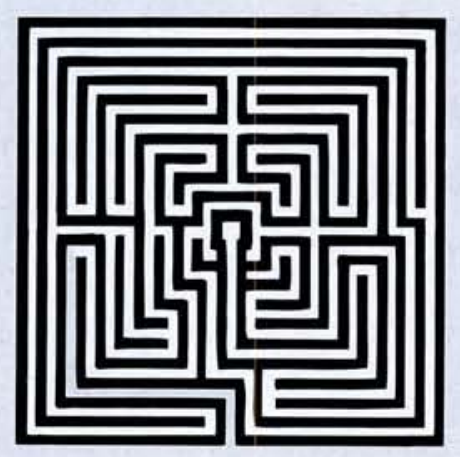

